\title{
Entre affect et apprentissage, la question de la perte. Inhibition psychique et inhibition cognitive 9
}

\author{
Between affect and training, the question of the psychic loss. \\ Psychic inhibition and cognitive inhibition
}

\author{
Michèle Benhaim ${ }^{\mathrm{a}, *}$, Nicolas Guérin ${ }^{\mathrm{b}}$, Jean-Jacques \\ Rassial $^{\mathrm{c}}$
}

\begin{abstract}
a Maître de conférence, habilitée à diriger des recherches, laboratoire de psychopathologie clinique et psychanalyse, faculté Saint-Charles, université de Provence Aix-Marseille 1, 3, place Victor-Hugo, 13001 Marseille, France

${ }^{\mathrm{b}}$ Psychologue clinicien, psychanalyste, postdoctorant, laboratoire de psychopathologie clinique et psychanalyse, faculté Saint-Charles, université de Provence Aix-Marseille 1, 3, place Victor-Hugo, 13001 Marseille, France

${ }^{c}$ Professeur, laboratoire de psychopathologie clinique et psychanalyse, faculté Saint-Charles, université de Provence Aix-Marseille 1, 3, place Victor-Hugo, 13001 Marseille, France
\end{abstract}

\section{Résumé}

Face à l'ampleur des demandes parentales ou scolaires relatives à «l'échec scolaire», ce texte présente l'intérêt d'articuler le concept d'inhibition en psychanalyse et celui d'inhibition en psychologie cognitive. Comment apprend-on? Quels sont les enjeux inconscients et cognitifs de la «faillite» de l'apprentissage ? De quelle logique de «renoncement» répond l'inhibition nécessaire à l'apprentissage et l'inhibition psychique pouvant peser dans le symptôme scolaire ? L'examen de ces logiques mènera à une proposition de prise en charge clinique associant la psychothérapie et un travail éducatif de remise en mouvement des processus intellectuels.

Mots clés : Inhibition ; Apprentissage ; Angoisse ; Perte ; Savoir

\section{Abstract}

In front of the extent of the parental or school requests relating to "the school failure", this text is of the interest to articulate the concept of inhibition in psychoanalysis and that of inhibition in cognitive psychology.

\footnotetext{
q Toute référence à cet article doit porter mention : Benhaim M, Guérin N, Rassial JJ. Entre affect et apprentissage, la question de la perte. Inhibition psychique et inhibition cognitive. Evol psychiatr 2009;74 (3).

* Auteur correspondant.

Adresse e-mail : michelebenhaim@voila.fr (M. Benhaim).
} 
How does one learn? Which are the unconscious and cognitive stakes of the "bankruptcy" of the training? Of which logic of "renouncement" answers inhibition useful for training and psychic inhibition being able to be transformed into a school symptom? The examination of these logics will carry out to a proposal for an assumption of responsibility clinical associating psychotherapy and an educational work of handing-over moving of the intellectual processes.

Keywords: Inhibition; Training; Anxiety; Loss; Knowledge

\section{Introduction}

L'intérêt clinique de se pencher sur la question des apprentissages à la fois du point de vue cognitif et du point de vue psychanalytique réside dans l'afflux de consultations d'enfants en âge scolaire, en centre médicopsychologique et en cabinet, le plus souvent accompagnés de leurs parents et reçus sur demande, voire sur injonction, de l'école. L'affaire nous est présentée sous les termes «d'échec scolaire», de «troubles de l'apprentissage», d'un «désintérêt général quant $\mathrm{au}(\mathrm{x})$ savoir(s)». Ces informations nous sont révélées sur fond de plaintes (narcissiques parentales), de dépits (dépressifs enseignants), plaintes assorties de revendications réparatrices immédiates mais d'emblée souvent sceptiques.

Là, soit les parents ont déjà soumis l'enfant à des investigations métriques de ses capacités intellectuelles (passation de tests d'efficience cognitive, bilans complets), soit ils annoncent, parfois clairement, qu'ils préfèrent envisager le point délicat sous l'angle d'un «blocage », entendre «symptôme », plutôt que sous celui d'une intelligence défectueuse.

Il nous paraît alors fructueux, au cœur de ces hésitations défensives, d'envisager globalement la question, d'emblée dans une dialectisation des enjeux, au sein de ce qui pourrait unir ces approches : quelle position du sujet est requise lorsqu'il se confronte au savoir? Le savoir étant ici à interroger non seulement comme savoir/connaissance mais aussi comme savoir inconscient, ces deux champs épistémiques ne se recouvrant pas. C'est donc la dialectique du sujet de la connaissance et du sujet du savoir inconscient qui est interrogée et interrogeable à l'occasion de ces demandes de consultations.

Les sciences cognitives et la psychanalyse nous enseignent en effet qu'il ne saurait s'envisager de sujet sans savoir (c'est pourquoi les expérimentations en psychologie cognitive se concentrent sur son évaluation en tentant de le saisir par le biais des processus d'apprentissage) et que le sujet se fonde lui-même d'un savoir duquel il s'excepte tout en s'y subordonnant (c'est ce qui légitime, d'une certaine façon, l'hypothèse de l'inconscient freudien).

Un double point de croisement entre ces deux disciplines peut ainsi, dialectiquement, se déduire: un point théorique selon lequel le sujet du savoir-connaissance (sujet cognitif) ou du savoir inconscient (sujet de l'inconscient) peut se définir comme un sujet logique; et un point clinique dans le constat partagé que, quand bien même intelligence cognitive il y a, le sujet ne l'utilisera pas, ou peu, ou mal, ou judicieusement, selon l'inscription de ses capacités intellectuelles dans sa position subjective. En outre, cette intelligence va-t-elle servir à nouer le symptôme et s'avère-t-elle être le lieu où les jeux symboliques prennent place et participent au développement? L'enfant va t-il être apte à cerner le plaisir qu'il peut en tirer et le mettre au service des apprentissages ou va t-il, dans une tentative de suppléer à sa douleur d'exister, en être encombré au point d'en ânonner répétitivement des bribes sous forme de stéréotypies, paraissant, dès lors, en faillite d'intelligence? 
Autrement dit, va-t-il pouvoir associer les processus requis pour apprendre et le désir de savoir?

Nombre d'enfants d'âge scolaire expliquent combien les savoirs les intéressent et comment leurs «absences » au moment de les restituer, leur fait énigme. D'autres réussissent des tests difficiles et semblent devenir presque sots dès lors qu'ils sont confrontés à une opération mathématique de base.

C'est dans cette confrontation que l'examen de la question à partir de notre double approche nous semble pertinent.

Elle visera à interroger le lien entre inconscient et cognition et ce, en se penchant plus précisément sur le concept d'inhibition. Nous verrons que, si ce terme ne se recoupe pas totalement selon les disciplines, l'inhibition a, de fait, partie liée, et avec le symptôme et l'angoisse nous dit Freud, et avec la maturation de la pensée et de l'intelligence nous précisent les postpiagétiens, en l'occurrence, Olivier Houdé [1].

Le nouage, par l'inhibition, du savoir inconscient et de la connaissance avait été maintes fois souligné par Freud, et ce jusqu'en 1938 lorsqu'il rappelait que «l'ultime fondement de toutes les inhibitions intellectuelles » ${ }^{1}$ relève de la «nature insatisfaisante en soi » de la pulsion sexuelle. Façon de dire que la fonction de la limite contenue dans l'inhibition re-présente une autre limite, cette fois non pas de contingence mais de nécessité, inhérente au réel du sexe qui fait la limite du savoir.

Or un des intérêts des recherches récentes postpiagétiennes est justement de mettre en exergue, à leur tour, le caractère de nécessité intrinsèque de l'inhibition cognitive.

En outre, c'est ce principe d'inhibition qui semble être à l'œuvre, négativement et/ou positivement dans l'acte d'apprentissage, acte qui engage simultanément la configuration neurobiologique et cognitive de l'apprenant et son bain affectif, culturel et social, c'est-à-dire son histoire subjective ${ }^{2}$.

Penser - cette histoire comme cette configuration en témoignent conjointement - nécessite la pensée, qu'un autre pense le sujet, pense pour le sujet, afin que s'établisse pour ce dernier le sentiment d'une continuité d'existence nécessaire à ce que l'objet puisse acquérir une qualité permanente, sur fond de présence/absence d'autrui dans un cas, et d'apparition/disparition de l'objet dans le second registre.

Dans une perlaboration de ces fonctionnements prend toute sa valeur et sa place la dialectique entre un sujet dit «personnel» et un sujet dit «indéfini réciproque ${ }^{3}$ distinction établie très tôt par Lacan, fondant véritablement une «théorie de l'esprit» consistante qui mobilise dans son fond le jeu des représentations mentales, du désir, et qui contribue à l'élaboration d'une «capacité de sollicitude ${ }^{4}$.

L'hypothèse que nous proposons d'étayer ici pose la question de l'inhibition dans son rapport à la perte et au manque : si du point de vue de la cognition, «penser, c'est inhiber», inhiber une structure concurrente - ce propos d'Olivier Houdé 5 montre bien la valeur positive qui est attribuée

\footnotetext{
1 Freud S. Résultats, idées, problèmes (1938). In: Résultats, idées, problèmes II, ([2], p. 288). Souligné par nous.

${ }^{2}$ La question pourrait d'ailleurs se poser de savoir «s'il est concevable que quelque chose soit du même ordre » entre cette inhibition qui affecte le sujet et la mise en fonction, dans le névraxe, d'une activité positive en tant qu'inhibitrice ; question qui ne sera pas traitée ici mais qui, soulignons-le, n'avait pas échappée en ces termes à Lacan (RSI, 10 décembre 1974).

3 Lacan J. Le temps logique et l'assertion de certitude anticipée. In : Ecrits ([3], p. 207).

4 Winnicott DWW (1963). Élaboration de la capacité de sollicitude (1963). In: Processus de maturation chez l'enfant, ([4], pp. 31 à 42).

5 O. Houdé [1] développe l'idée que «penser, c'est inhiber », c'est-à-dire que tout acte de pensée met en jeu plusieurs opérations (inhiber les stimuli dangereux ou inadéquats, activer les stimuli non dangereux mais pertinents, enfin inhiber
} 
ici à l'inhibition - du point de vue psychopathologique l'inhibition n'est jamais très éloignée du symptôme, ce qui pourrait lui conférer un statut négatif, au sens de «morbide ». Pourtant, si, pour la psychanalyse, le symptôme ne se réduit pas au pathos, il convient d'ajouter que la présence de l'inhibition affective n'entache pas forcément les apprentissages, mais à quel prix ?

Nous avons rencontré des enfants brillants scolairement mais en grande difficultés relationnelles, voire, présentant de véritables phobies scolaires.

Chaque nouvelle conquête cognitive créerait une complexification supplémentaire obligeant l'enfant à inhiber, selon la tâche à accomplir, une de ses compétences. Ce courant de pensée remet en cause la linéarité du développement, par «superposition» de structures cognitives de plus en plus complexes (macrogénétique de Piaget). Il existerait en permanence une compétition entre les différentes potentialités que possède l'individu pour résoudre une tâche. Cette théorie définit l'intelligence comme la capacité à inhiber de la manière la plus opportune possible certaines de ses acquisitions.

C'est pourquoi nous argumenterons dans un champ, puis dans l'autre, autour du concept d'inhibition afin de montrer comment s'articulent l'inhibition «affective » qui peut aller jusqu'à embarrasser le sujet, et l'inhibition cognitive nécessaire à l'adaptation, c'est-à-dire à la construction de l'intelligence, possible, parfois trop possible (comme dans certaines formes d'autisme).

Articuler le double aspect de l'inhibition mis en lumière par ce souci de l'appréhender à partir d'un double champ conceptuel a, de plus, l'avantage de souligner la duplicité du symptôme dit «scolaire », ici logé et à déchiffrer sur l'Autre scène du savoir.

Il semble que la stratégie que recouvre «penser c'est inhiber» semble faillir chez certains enfants qui n'auraient pas suffisamment investi la pensée du temps de la petite enfance : le maintien d'un certain plaisir à penser fera défaut comme apport narcissique au moment où l'intensité des capacités cognitives confronte l'enfant à un doute. Freud [5] faisait de sa pulsion épistémophilique une tentative d'emprise sur le monde et montrait que l'effroi devant un tel mouvement pouvait susciter une inhibition de la pensée, mécanisme de défense invalidant. La naissance de la pensée s'origine dans la relation primordiale. Dans le champ des excès (de haine ou d'amour maternel en l'occurrence) se loge l'impossibilité d'investir la représentation. Nous retrouverons ces aspects en jeu dans l'exemple clinique que nous développons plus bas. Car, bien entendu, c'est la clinique qui nous engage à tenter cette double approche, les « rééducateurs » nous faisant très vite part des limites de leur travail dans le traitement du symptôme dit «scolaire».

\section{L'inhibition et la cognition}

Olivier Houdé, revisitant «la psychologie de l'enfant», estime que les stades piagétiens sont à repenser au vu des nouveaux résultats expérimentaux obtenus à partir d'observations de bébés et de la confirmation de ces résultats par les neurosciences.

Si nous le suivons sur ce chemin de devoir décrire le développement de l'enfant à partir d'une approche temporelle discontinue car empreinte d'erreurs, de régressions cognitives, de «vagues qui se chevauchent» [6] plutôt que, comme l'envisageait Piaget [7], caractérisée par une suite linéaire et cumulative d'acquisitions intellectuelles soumises (tout en témoignant de son efficience) au stade auquel l'enfant est parvenu, nous pouvons considérer qu'Houdé fait du temps chronolo-

les schémas non dangereux, non pertinents). Certains enfants échoueraient dans les apprentissages parce qu'ils n'auraient pas activé une de ces opérations. 
gique piagétien une sorte de temps logique ${ }^{6}$ où l'enfant ne saurait se contenter de «voir » l'objet mais devrait faire l'effort d'en «comprendre » le traitement pour «conclure » à sa permanence, principe au fondement de la construction de la réalité. Chaque terme de cette temporalité logique, instant de voir, temps pour comprendre et moment de conclure, ne s'inscrit pas dans une continuité stricte et idéale mais se fonde d'une discontinuité faite de scansions suspensives nécessaires qui peuvent parfois se manifester sous la forme d'une inhibition du développement intellectuel. La dialectique entre sujet du savoir inconscient et sujet de la connaissance est envisageable ici notamment dans le fait que l'effectivité du temps pour comprendre est pleinement compatible, voire rend nécessaire, l'inhibition simultanée des acquisitions dans tel champ de connaissance. Dans cette mesure, les dernières recherches postpiagétiennes ne définissent pas l'inhibition en termes de déficit mais l'inscrivent, au contraire, au cœur du procès logique du sujet.

Si parallèlement, nous appliquons la même modification aux stades freudiens, nous tombons, non plus sur une description, en termes psychosexuels, de l'évolution de l'enfant mais sur une sorte de psychogenèse de l'Autre, la constitution de ce dernier lieu et des figures qui l'incarnent tour à tour, (mère, parents, Autre sexe), participant en la fondant, de la construction de l'altérité, que l'autre soit présent et perçu ou bien qu'il s'absente et se représente.

Le développement cognitif visait, pour Piaget, à la synthèse des processus d'assimilation et d'accommodation, dont l'aboutissement est l'adaptation, synonyme d'intelligence, et ce, à travers l'action que l'enfant pouvait exercer sur le monde.

La dynamique qu'Houdé propose pour renverser cette positivation absolue du mouvement évolutif, en termes d'activation/inhibition dans leur dimension adaptative, ouvre l'opportunité d'un dialogue avec la psychanalyse : d'une part, en rappelant les écueils nécessaires à la construction subjective, d'autre part, en permettant d'associer, sans les superposer, les processus primaires et les processus secondaires freudiens à la dynamique activation/inhibition cognitive.

Rien n'est plus difficile que d'inhiber sa propre croyance, rien n'est plus douloureux que de renoncer à la toute-puissance infantile. L'inhibition ne recouvre pas le deuil mais le travail psychique nécessaire à leur mise en œuvre requiert des élaborations comparables en termes de renoncement et de perte.

Il semblerait qu'en termes de développement et de subjectivation, ces «pertes » impossibles engendrent des pathologies et/ou des dysfonctionnements touchant au symbolique. Si l'on s'appuie sur les processus primaires et secondaires en les articulant aux processus d'activation/inhibition, on peut penser que les altérations qui peuvent en entraver le bon déroulement touchent aux représentations et s'enracinent dans des déséquilibres entre liaison et déliaison.

Un petit exemple clinique illustre ce point : il s'agit d'un petit garçon de neuf ans supportant le diagnostic de «troubles de l'apprentissage et dysphasie». Cet enfant a la particularité de ne pouvoir écrire les lettres autrement qu'en les dessinant en utilisant des formes géométriques (bâtons, ronds ....). Ce phénomène clinique relève, nous semble t-il d'une inhibition tant pour les sciences cognitives (inhibition de l'apprentissage de l'écriture) que pour la psychanalyse («effet d'arrêt » qui résulte d'une intrusion de l'imaginaire - formes géométriques - dans le symbolique $-l^{\prime}$ 'écriture -$)^{7}$. Cette inhibition s'avèrera, contrairement aux apparences, et venant appuyer les hypothèses d'Houdé, un temps nécessaire à une autre acquisition: la possibilité pour ce petit garçon de se séparer de sa mère sans trop d'angoisse (écrire, oui, mais «pas tout à fait», pas

\footnotetext{
6 Voir l'article de Lacan « le temps logique et l'assertion de certitude anticipée », ([3], p. 207) où est décrite la temporalité du sujet, son essence sociale et logique à partir d'une dialectique de temps distincts et de scansions nécessaires.

7 J. Lacan, RSI 10 décembre 74, inédit.
} 
encore tout à fait) et d'autre part, elle mettra en lumière (côté psychanalyse) l'indice d'un désir que l'inhibition occulte et indique à la fois ${ }^{8}$.

En effet, ce que mit à jour le suivi thérapeutique de ce petit garçon, c'est une difficulté de passer d'un tracé imagé (les formes géométriques relevaient, dans son discours, de «dessins ») à un tracé symbolique. Comme si le problème résidait dans une quasi-impossibilité de détachement de ce qui existait pour lui dans sa petite enfance : le champ oral du parler que cet enfant tentait de retranscrire tel quel, ne pouvant prendre à son compte l'écrit, c'est-à-dire ne pouvant assumer le passage de l'image au mot.

Ce qui nous mit sur cette piste et qui témoigne de la nécessité de travailler à plusieurs sur les deux registres que nous tentons de faire dialoguer (psychanalyse et sciences cognitives), ce sont les propos dont nous fit part l'orthophoniste. En effet, celle-ci s'étonnait de l'effort physique que semblait mettre en œuvre l'enfant à partir de tout son corps et des mouvements de lèvres qui accompagnaient cet effort. Nous avons alors pu faire l'hypothèse de l'aspect contraignant que pouvait représenter le tracé d'écriture pour ce petit garçon, le désir de contrôle que pouvait recouvrir cette nécessité de «parler» pour écrire et, ainsi, dans ce double mouvement, la difficulté à céder du côté de la toute-puissance infantile avec ce bénéfice de conserver la mère à ses côtés.

Si nous avions repéré la douleur à l'œuvre dans le processus de séparation, ne faisant bien sur pas écrire l'enfant, sans l'orthophoniste nous n'aurions pas eu accès à cette observation relative à l'oralité.

À partir de ces échanges, nous avons mieux appréhendé certains aspects de clivage chez cet enfant: notamment le fait qu'il s'exprimait extrêmement bien mais que son discours semblait dénué d'émotions; nous les avons «lus» à partir de notre double approche: cet accrochage aux formes imagées plutôt qu'aux symboles purs signait une sorte d'intellectualisation de sa souffrance et avait pour bénéfice de lui en éviter la psychisation (symbolisation/subjectivation) douloureuse.

La suite de la thérapie consista alors à permettre à l'enfant d'inscrire dans sa subjectivité sa difficulté d'apprentissage et d'en exprimer un ressenti propre. Jusqu'alors, il repérait bien la difficulté dans l'entourage familial et scolaire mais il résistait à se l'approprier.

Si l'écriture, symbolique, relie et sépare à la fois, pour ce petit garçon, écrire «presque »a pu permettre, en inhibant le versant séparation, de se maintenir exclusivement dans le lien.

À notre tour alors de faire part de ces données d'un registre narcissique à l'orthophoniste qui a pu entreprendre plus précisément un travail à partir de l'engagement du corps de l'enfant, dans des propositions de tracés libres à partir desquels l'enfant devait évoquer ses ressentis. Le tout sous le regard étayant de l'orthophoniste, alors transférentiellement garante d'un non-effondrement subjectif.

Nous savons qu'économiquement, les processus primaires que Freud situe dans le système inconscient se caractérisent par le libre écoulement de l'énergie psychique et par le libre glissement de sens. Pour ce, l'inconscient utilise des mécanismes tels que le déplacement et la condensation pour assurer le mouvement d'une représentation à une autre. Il en va ainsi de l'activation lors de la sélection d'informations que doit opérer le sujet de la connaissance d'un point de vue cognitif s'il veut résoudre une tâche.

De la même façon qu'un appareil psychique ne saurait se concevoir sans une limitation de ce libre écoulement d'énergie, les processus d'apprentissages ne peuvent s'envisager sans un frein à leur excitabilité.

\footnotetext{
${ }^{8}$ C'est ce que Lacan dit de la fonction de l'inhibition dans son séminaire sur l'Angoisse.
} 
C'est pourquoi Freud adjoint aux processus primaires des processus secondaires qui, à l'inverse des premiers, ont la particularité de voir l'énergie psychique soumise à un contrôle, sous l'égide du principe de réalité. Freud loge ces processus dans le système préconscient-conscient et en fait le véritable socle de la pensée logique.

Ainsi se poursuit notre analogie avec l'inhibition comme mécanisme fondamental impliqué dans la résolution d'une tâche cognitive.

Les processus secondaires contrôlent une pensée qui serait, sinon, trop libre. L'inhibition contrôle l'afflux de connaissances déjà acquises mais qui, ici, si elles n'étaient pas inhibées, entraveraient par leur inefficience, le raisonnement.

Un défaut d'inhibition comme une faillite des processus secondaires attaquent les mouvements représentatifs. L'exemple que nous venons d'exposer montre que là où la représentation est attaquée, l'organisation du lien à l'objet rencontre un écueil. Comme si ce défaut d'inhibition cognitive court-circuitait l'élaboration censée permettre l'émergence du désir sur le plan affectif et celle de l'intentionnalité sur le plan intellectuel.

Cliniquement, ces difficultés se traduisent souvent par un rapport au savoir fait, pour le coup, d'inhibitions manifestes causées, en quelque sorte, par un défaut d'inhibition latent pouvant mener à un véritable désinvestissement de type parfois phobique, donnant lieu à des discours pauvres en représentations. Or ce qui ne peut se représenter est bien ce qui se lie primairement. Par ailleurs, la permanence de l'objet, cet objet qu'il faut retrouver hors perception en inhibant une croyance (du lieu où on l'a perçu antérieurement par exemple) repose sur l'intégration de sa perte et convoque sa représentation interne. L'inhibition manifeste porterait, dans notre exemple, sur un lien relationnel trop primaire, l'inhibition cognitive impossible sur la croyance que le mot pourrait imager et non représenter la chose, (les formes géométriques fonctionnaient pour le petit garçon, rappelons le, comme une tentative de dessiner ce qui aurait du être écrit). L'on pourrait dès lors supposer un effet «symboligène », voire épistémogène, de l'inhibition et du processus de décroyance qui lui est corrélatif.

À défaut d'inhiber la croyance reposant sur la perception, une sorte de formation psychique se crée immédiatement telle une perception.

L'inhibition cognitive nécessite un temps pour comprendre, sinon, figé dans l'instant de voir, l'enfant laisse s'écouler sa croyance spontanément et conclut dans une résolution de tâche erronée, au nom, probablement, de la recherche d'une satisfaction immédiate.

Ce temps pour comprendre qui fait, pourrait-on dire, passer le sujet du spéculaire (instant de voir) au spéculatif, fait aussi appel aux processus secondaires qui mettront le sujet sur des voies détournées de satisfaction, via l'effectivité des représentants de mots (et non plus de choses).

Olivier Houdé, fidèle à Jean Piaget pour lequel la construction la plus fondamentale était celle de l'objet, va examiner lui aussi le cheminement nécessaire à l'enfant pour parvenir à un objet permanent. Il va, pour cet examen, et à partir des mêmes expérimentations assorties des mêmes erreurs de localisation, (erreur A-nonB) ${ }^{9}$, se pencher sur trois traitements possibles de l'objet: son traitement quantitatif (le nombre), qualitatif (la catégorisation), les hypothèses ou déductions que l'objet supporte (le raisonnement).

Nous retiendrons ici deux points de conclusion :

\footnotetext{
${ }^{9}$ L'erreur dite $\mathrm{A}$ non $\mathrm{B}$ consiste, pour un bébé de 9-12 mois à retourner chercher dans un endroit $\mathrm{A}$, utilisé au préalable comme cache, un objet caché sous ses yeux dans un endroit B.
} 
- «je pense donc je me trompe»; les erreurs que l'enfant fait jusqu'à l'âge de 12 mois (mais qu'il ne faisait pas à $4 / 5$ mois, et que Piaget traduisait par un défaut de permanence de l'objet) sont, en fait, des erreurs que l'adulte continue de faire ${ }^{10}$;

- «je pense, donc j'inhibe »; il existe une logique de l'erreur, non par défaut de permanence de l'objet, mais par défaut de capacité d'inhibition d'un savoir présent mais inopportun à ce moment là et dans cet espace (mental) là ${ }^{11}$.

Ainsi, pour être intelligent, il faut prendre le temps pour comprendre (temporalité logique et inconsciente qui correspond aux processus secondaires du point de vue psychique, recouvrant ce que les cognitivistes nomment la reconstruction à travers le langage), ne pas en croire ses yeux d'une certaine façon, «évider l'évidence » disait Lacan, ce qui pourrait correspondre au fait d'avoir la capacité d'inhiber une stratégie acquise: autrement dit, pour raisonner, il faut quitter le champ perceptif et intuitif: pour être intelligent, il faut accepter de perdre. Pour écrire, il faut accepter de se séparer.

En ce sens, l'examen attentif des déclinaisons de l'inhibition et de la perte au sein des trois formes d'assujettissement à la structure du langage que distingue la psychanalyse (à savoir, la psychose, la névrose et la perversion) mériterait à lui seul une étude. Celle-ci pourrait ainsi restituer «l'ordre des raisons » qui soutient trois occurrences différentes d'intelligence psychotique, névrotique ou perverse et ce, sans considération déficitaire pour l'une ou l'autre de ces positions subjectives. L'on sait, par exemple, que la psychose de certains sujets (comme le mathématicien Georg Cantor notamment) leur permet d'explorer les fondements de la structure du langage (fut-il celui des mathématiques) jusqu'à pouvoir en extraire ses propres limitations internes. Or cette forme d'intelligence n'est pas non plus sans faire intervenir ce que certaines psychoses cliniques révèlent par le biais d'une construction délirante par exemple, à savoir «l'inhibition » (au sens postpiagétien) d'une croyance (Unglauben) en un savoir déjà là, traditionnel et incarné par quelques figures d'autorité qui embarrasse d'ordinaire le renouvellement du rapport du sujet au savoir. Dans cette mesure, cette «inhibition » psychotique de la croyance en un savoir présupposé et figé au lieu des «vérités éternelles » est conforme à l'acte de l'homme de science qui, pour renouveler le savoir de la science, doit franchir le seuil (inhiber) de la croyance en un savoir préexistant à découvrir - franchissement ou inhibition qui l'autorise à inventer. Et il faut bien le reconnaître, l'intelligence psychotique est loin d'être impropre à l'invention, du moins si l'on veut bien dégager la psychose de sa gangue morbide.

D'un point de vue général, l'on peut avancer que de la même façon que les processus primaires doivent limiter la saturation des processus secondaires et que les processus secondaires doivent limiter la saturation des processus primaires, dans la jungle des compétences précoces du bébé ou de l'enfant, voire même de l'adulte, où les savoirs se construisent de façon non pas linéaire mais décalée, un mécanisme doit pareillement limiter la saturation de ce foisonnement : cet élément de l'adaptation cognitive est l'inhibition.

\footnotetext{
10 Olivier Houdé a mis en évidence que le développement de l'intelligence jusqu'à l'âge adulte est jalonné d'erreurs de logique, de biais perceptifs non prédits par la théorie piagétienne. Plutôt que de suivre un plan qui mènerait, sans retour en arrière, du stade sensorimoteur à l'abstraction, l'intelligence de l'enfant avance de façon beaucoup plus biscornue.

11 Pour Olivier Houdé, «penser, c'est inhiber» traduit aussi la capacité à ressentir certaines émotions nécessaires pour inhiber des comportements inadéquats.
} 


\section{L'inhibition, le symptôme et l'angoisse}

La question de la pensée, nous venons de le voir du point de vue cognitif, est indissociable de celle de l'inhibition. Il faut inhiber, pas pour rien, pour apprendre. Se développer, c'est apprendre à inhiber, inhiber des stratégies en compétition.

Que devient cette perte nécessaire du point de vue psychique et, plus avant pour ce qui concerne, non plus l'accès au savoir, mais son malaise et ses «troubles », du point de vue psychopathologique?

Dans son séminaire de 1962-63 consacré à l'angoisse [8], Jacques Lacan, situant la triade freudienne, inhibition, symptôme et angoisse [9] dans une matrice à double entrée, dite «tableau de l'angoisse », place et définit l'inhibition comme un point structural dans la constitution du désir et de sa forme évolutive. L'inhibition n'est ainsi pas abordée d'un point de vue déficitaire ou simplement pathologique. Elle est au contraire la marque et, à la fois, le masque d'un désir. Si Monsieur Untel, nous dit Lacan, a la crampe de l'écrivain, c'est qu'il érotise la fonction de la main. Ici l'inhibition de l'écriture est le colophon du désir. De même lorsque, quelques années auparavant, Lacan réinterprétait, à la suite de Freud, l'inhibition d'Hamlet à tuer Claudius ${ }^{12}$. Là où Freud voyait déjà dans cette inhibition la marque de l'interdit de la réalisation d'un désir œdipien (tuer le Père à travers la figure de Claudius), Lacan y verra plus radicalement la condition tragique du désir comme tel : le sujet inhibe la réalisation de son désir pour conserver son désir. Car réaliser son désir, c'est en même temps l'abolir.

L'inhibition ne sera donc pas dévalorisée par Lacan. Il tentera au contraire de la resituer et de la redéfinir, et ce jusqu'à la fin de son enseignement où, situant la triade freudienne l'inhibition, le symptôme et l'angoisse, par rapport à sa triade à lui, l'imaginaire, le symbolique et le réel, il finira par élever l'inhibition à la dignité d'un nom du père en en faisant une nomination imaginaire en tant qu'elle est ce qui fait barre au niveau de l'imagination ${ }^{13}$.

Ainsi, si l'inhibition n'est pas incompatible avec le désir, elle ne saurait non plus l'être avec cette figure particulièrement problématique du désir qu'est le désir de savoir. En ce sens, il n'est pas paradoxal de penser que telle inhibition scolaire dans l'acquisition d'une connaissance soit en fait révélatrice de la manifestation du désir de savoir qui lui, ne l'oublions pas, est toujours lié à la dimension du sexuel.

Lorsque le petit garçon que nous évoquions plus haut a pu se résoudre à dire ce qu'il ressentait au moment d'écrire, il nous fit part d'une sorte de douleur corporelle due à une crispation, non seulement de sa main, mais de son corps tout entier et il put exprimer l'épuisement qui résultait de cette tension. Ce point n'est pas sans nous évoquer «la crampe » soulignée par Lacan.

Freud faisait de l'inhibition une limitation normale fonctionnelle du moi, c'est le freinage à l'œuvre lorsqu'il s'agit, pour l'enfant, de nous dire où se trouve l'objet caché quand il a été déplacé du lieu où il l'a perçu au départ.

Certes, l'inhibition peut toucher au symptôme lorsque, notamment, le moi ne se voit plus limité mais perturbé au point que l'action elle-même ne peut avoir lieu.

Le symptôme scolaire du petit garçon témoignait-il, à travers cette «perturbation » d'une soumission à un Idéal du Moi trop exigeant?

Plus la signification sexuelle s'accroît, plus la limitation vire à la perturbation. Peut-on faire alors l'hypothèse que pour le très jeune enfant, réussir la tâche, trouver l'objet repose sur le

\footnotetext{
12 Lacan J. Le désir et son interprétation (1958-59), Inédit.

13 J. Lacan. RSI, séminaire inédit du 13 mai 1975.
} 
refoulement d'un désir interdit? Ne plus le trouver, lorsque l'enfant est plus grand, et ce, pour un temps seulement, s'articulerait alors à la signification sexuelle que revêt alors la tâche et entraînerait le moi à renoncer, temporairement, à sa fonction "pour ne pas avoir à entreprendre un nouveau refoulement» Le cours de ce refoulement peut être repris dans un second temps, ce dont témoigne la progression cognitive qui fait suite à la régression.

Cette hypothèse psychanalytique aiderait à lever l'énigme développementale que les cognitivistes postpiagétiens interrogent à travers le concept de «défaut d'inhibition »en place de non-acquisition pure et simple.

L'inhibition, dont on perçoit bien la dimension positive en psychologie cognitive, revêt une nécessité semblable quand Freud la définit comme un commencement de sublimation. Il n'a de cesse de rappeler, tout au long du chapitre qu'il lui consacre dans Inhibition, symptôme et angoisse, que l'inhibition ne signifie pas nécessairement quelque chose de pathologique. En psychologie cognitive, cette limitation normale d'une fonction est nécessaire à la réussite intellectuelle recouvrant la construction de la permanence de l'objet. Dans un champ comme dans l'autre, ce qui pourrait signifier que l'inhibition a touché au symptôme, c'est que l'action soit, non plus freinée, nécessairement freinée, mais empêchée, c'est-à-dire d'ores et déjà soumise à l'angoisse.

Sur l'axe de la difficulté, que Lacan déploie avec sa matrice [8], embarrassé d'une perception en trop, l'enfant peut, en effet, résoudre le dilemme en se débarrassant de «cette forme légère de l'angoisse », c'est-à-dire en formulant une réponse erronée.

Au contraire, si l'affect se modifie, cette fois sur l'axe du mouvement, et plonge l'enfant dans l'émoi, écrasé d'impuissance, il se tait, ne peut, d'une certaine façon, un peu schématique, activer le processus inhibiteur pas plus que le processus activateur. Schématique parce que le modèle binaire activation/inhibition est, bien sûr, impropre à la psychanalyse. Il convient à la psychologie du développement en tant qu'elle emprunte un modèle, une métaphore neuronale.

En termes cliniques, cependant, il n'apparaît pas comme réducteur, mais nous aide, comme il en ressort de l'exemple du petit garçon, à la fois dans l'orientation de la psychothérapie et dans la mise en place d'exercices d'apprentissage.

Ainsi, au cœur de cette double approche, pouvons nous proposer que l'inhibition se situe endeçà de l'empêchement ; en outre, à l'intérieur même du processus adaptatif, activation/inhibition, ces deux mouvements sont opérants s'il se trouve juste ce qu'il faut d' "empêchement» pour inhiber, freiner, c'est-à-dire ne pas activer exclusivement et à tout crin, juste ce qu'il faut pour que l'angoisse ne soit pas seule moteur de désir.

Passer en quelque sorte de «presqu'écrire » à «écrire » mais pas au prix d'un effondrement psychique.

C'est en deçà de l'émotion que l'inhibition peut recouvrir positivement cette énergie mise au service de la cognition et non de l'affect pur (l'angoisse ?).

Voici qui nous importe grandement si nous rappelons que l'inhibition représente la condition même de la représentation, en cognition comme dans les structures des névroses.

Enfin, complétons ces figures hypothétiques par l'apport cognitif lorsqu'il fait de l'émotion le précurseur de l'élaboration d'une théorie de l'esprit ${ }^{14}$, théorie à valeur hautement symbolique, s'inscrivant en rupture avec la pensée égocentrique piagétienne qui voyait son activation mise au seul service de la satisfaction. Ajoutons sur ce point que les sciences cognitives, faisant récemment une plus large place à l'émotion, bénéficieraient des apports de la psychanalyse en la matière qui,

\footnotetext{
${ }^{14}$ La théorie de l'esprit est la capacité à attribuer des intentions, des croyances, des désirs ou des représentations mentales à autrui et à soi-même.
} 
certes, n'est pas une théorie des émotions mais qui, avec les apports de Lacan en 1962 ([8], p. 20-21), problématise cette notion et évite l'écueil qui consiste à la réduire à un terme générique imprégné du sens commun.

\section{La question de la perte}

La mise en place et le rapport à l'objet (au sens de l'objet dit permanent en psychologie du développement, et au sens de l'objet de la dyade freudienne, moi/objet) éclairent le rapport au manque au travers du symbolique et nous renseignent sur la singularité du sujet à entrer (ou à en être empêché) dans le «code». Sur un système de références symboliques repose la maturation des capacités cognitives de l'enfant.

Comment l'enfant prend-il conscience de l'existence de l'objet ? C'est-à-dire comment réalise t-il que l'objet continue d'exister hors de la perception qu'il peut en avoir? Comment supporter l'absence de la mère ? Comment peut-elle être suffisamment «présente sur fond d'absence » pour que la question de sa disparition potentielle ne parasite pas les processus d'apprentissage?

C'est la toute-puissance infantile et l'illusion créatrice du bébé qui lui permettent d'avoir l'assurance de retrouver l'objet là où il lui a attribué une place absolue et quand il le souhaitera. Ce qui lui fait vivre comme perte imaginaire, atteinte narcissique, la simple reconnaissance d'un manque symbolique structurel déjà inscrit dans la fonction de représentation.

L'erreur A-nonB révèle le décalage entre l'imaginaire, aussi bien image du corps qu'image du monde, dite réalité et la logique cognitive ${ }^{15}$, orientée par l'essai, confronté à la limite de l'erreur et de l'exception, trace du manque, de symboliser le réel. Ce qui prime ici, sous l' «erreur », c'est la toute-puissance absolue de l'enfant pas tout à fait prêt à renoncer à être l'objet qui comble la mère ou, autrement dit, pas tout à fait prêt à consentir à ce que la structure du langage s'établisse sur une place vacante qu'aucun objet absolu, sauf un fétiche, ne viendrait saturer. Et que c'est là la seule condition à ce que l'enfant re-trouve les objets si au moins un a été perdu ou inhibé à sa place.

Ne prime donc pas l'erreur cognitive elle-même : preuve en est la «logique » qu'elle comporte et qui repose sur ce que Olivier Houdé pointe de la nécessaire inhibition à l'origine de toute nouvelle acquisition.

$\mathrm{Si}$, comme le souligne Freud, «mémoire et conscience s'excluent ${ }^{16}$, 1'accès à la représentation doit en passer par une perte, comme reconnaissance du manque: c'est ce dont témoigne, en cognition, le nécessaire tri dans les acquis des données qui serviront aux acquisitions suivantes, l'inhibition. Perdre un savoir devenu encombrant, embarrassant.

Aller vers B nécessite de renoncer, de lâcher, de perdre A et «le jugement constitue un processus psychique que seule une inhibition venue du moi rend possible $»^{17} \ldots$

Ne pas lâcher A mène à l'échec d'avoir interrompu la mobilité du processus de pensée, sauvant ainsi cette dernière de l'angoisse.

La compétition cognitive à l'œuvre n'est pas sans nous évoquer la lutte que se mènent les instances psychiques freudiennes et la résistance qui insiste lorsque la levée du refoulement point. Apprendre, c'est aussi prendre le risque de perdre : c'est le prix à payer du passage d'une logique

\footnotetext{
15 Processus flexible où la logique de l'adaptation prend le pas sur celle de la raison en se fondant sur le travail cognitif opérant dans la distribution et le regroupement d'objets et dans la catégorisation des «sens» de ces objets pour leur construire des «natures ».

16 Freud S. «Lettres à Fliess ». In: Naissance de la psychanalyse, ([10], p.154).

17 Freud S. Esquisse d'une psychologie scientifique (1895). In: Naissance de la psychanalyse, [10].
} 
du savoir inconscient à une logique de l'autre savoir, d'une logique des processus primaires à une logique de signification.

La perte se signerait d'un renoncement : renoncement à une fonction, nous dit Freud, à une stratégie, avance Houdé. Sous un angle comme sous l'autre, c'est la tendance générale à cette renonciation qui ferait symptôme.

Que l'on situe tour à tour la question de l'inhibition comme un conflit moi/surmoi ou moi/ça, peut-on s'avancer à y lire une analogie avec ce qui entrerait en conflit entre l'inhibition et l'activation et qui mènerait à l'«erreur»?

Pourquoi, n'inhibe t-on pas ? Pourquoi l'enfant ne peut-il se résoudre à perdre ses théories sexuelles infantiles au profit d'un apprentissage? N'est-ce pas précisément au carrefour du savoir inconscient et du savoir/connaissance que se logeraient les «troubles » de l'apprentissage ? Les uns faisant les frais des autres.

Chez un enfant qui n'apprend pas, il existe néanmoins toujours du savoir, une théorie qui lui est propre et qui, en interne, a sa cohérence. À ce carrefour, nous devons cheminer avec l'enfant au travers des méandres complexes que cette théorie emprunte souvent.

Du point de vue psychique, le moi se trouve limité dans sa fonction, soit par mesure de précaution, soit en raison d'un appauvrissement d'énergie.

Si la stratégie cognitive est mise en péril, effacée, désactivée au lieu d'être inhibée, on peut penser que lorsque le sujet en a besoin, elle n'est plus à sa disposition. Aussi, si cette stratégie devient nécessaire au cours d'un processus d'apprentissage, on assiste à une défaillance cognitive, autrement dit à un empêchement.

Reste à savoir si, selon les sujets et les moments logiques de leur suivi psychologique, cet empêchement peut s'élever au statut d'un symptôme, au sens analytique du terme, c'est-à-dire au sens d'une formation de l'inconscient. Car si tel est le cas, le symptôme est le lieu du retour du refoulé et donc du retour de la stratégie cognitive «refoulée ». Dans cette mesure, et dans cette mesure seulement, l'on pourrait vérifier que, pour un sujet, le symptôme est sa stratégie dans le sens où il pourrait prendre sa valeur d'usage pour le sujet, un savoir y faire singulier avec le savoir en quelque sorte, là où il n'y avait, auparavant, qu'empêchement.

L'inhibition de la curiosité sexuelle va-t-elle se renverser en curiosité intellectuelle ou, au contraire, lui faire courir le risque de se figer à son tour? Un élément de réponse se loge peut-être dans la qualité de cette inhibition dont le degré d'intensité retentirait sur la capacité à appréhender le monde, c'est-à-dire, à apprendre.

Une perte trop douloureuse sidère la pensée, ce qui limite lourdement désir et plaisir, les deux mouvements à engager et à soutenir au cœur des apprentissages.

$\mathrm{Si}$, pour compter, par exemple, il nous faut supporter la discontinuité dans la conservation, certains enfants ne peuvent s'y risquer tant le danger d'un monde où les êtres et les objets peuvent disparaître (non-permanence) est grand. Or l'unité de base cognitive, c'est la permanence de l'objet.

Nous venons de tenter de distinguer les modèles postpiagétien et psychanalytique car ils ne sauraient se superposer. Néanmoins, oublier, éviter, inhiber, renoncer, refouler? Entre renoncement et refoulement, ne pouvons-nous situer l'inhibition? Ainsi se résoudrait la perte dans son aspect de nécessité développementale.

\section{Conclusion : incidences cliniques}

Nous avons montré comment Olivier Houdé nous engage à reconnaître à l'inhibition une histoire cognitive propre : Se développer, c'est (aussi) inhiber. 
Les neurosciences valident largement cette hypothèse en faisant porter l'inhibition sur ce qu'elles nomment «savoirs dangereux »... (en neuroscience, le défaut d'inhibition cognitive relèverait d'un défaut d'inhibition de «schèmes dangereux » liés à des savoirs expérientiels et implicites du bébé, situés au niveau des structures de mémorisation d'expériences passées).

Olivier Houdé montre que dans cette histoire cognitive, la question des rapports entre rationalité et inhibition se pose dès la genèse de l'unité de base du réel cognitif qu'est l'objet dans sa permanence. Les expérimentations actuelles à l'origine de ses démonstrations reposent sur les mouvements oculomoteurs des bébés qui, soumis dès quatre/cinq mois à cette activité témoignent de l'existence d'une unité et d'une permanence précoce de l'objet. Ainsi, lorsque l'enfant échoue dans une épreuve de catégorisation logique, il échoue en raison d'un défaut d'inhibition d'un mode de fonctionnement concurrent; lequel défaut est, comme tel, logique.

N'oublions pas l'insistance de Freud à espérer «un substrat biologique » à sa «phase de latence», celle, en l'occurrence qui recouvre l'âge scolaire. Mais, soulignons-le, notre position quant à l'activité cognitive consciente et inconsciente n'est pas naturaliste. Car l'espoir freudien susmentionné est moins l'aveu d'un biologisme sous-jacent à la théorie analytique que la délimitation d'obstacles intrinsèques à la théorie, obstacles qui, conformément à la notion d' «obstacle épistémologique » promue par G. Bachelard, ne constituent pas seulement des points de stagnation ou d'inertie quant au savoir, mais dont le repérage et l'interrogation participent à la relance de la théorie. C'est ainsi que Roland Gori rappelle que «la démission [ponctuelle de Freud] au profit de la biologie résulte d'une position d'abstention en matière de causalité, abstention qui vient heuristiquement se constituer au bord même de ce qui fonde la psychanalyse » ([11], p. 24).

Sur le plan qui nous intéresse, la clinique analytique en tant qu'elle croise les sciences $\operatorname{cognitives}^{18}$, que faire de cette double tentative de compréhension, au-delà de l'histoire singulière et du discours du sujet pris dans ces difficultés?

Ce qui nous engage au dialogue interchamps conceptuels et à une approche clinique large, incluant l'approche développementale et l'aspect intersubjectif, repose sur la longueur des processus mis en mouvement lorsqu'un enfant présente un «échec scolaire » et sur les conséquences liées à cette lenteur: c'est le «moi » qui se rend à l'école, le «symptôme»s'enlise et l'enfant est «orienté », bien entendu en son désavantage puisque c'est souvent d'exclusion dont il s'agit, cette orientation grevant alors lourdement son avenir.

L'idée qui sous-tendrait la question thérapeutique pourrait relever du passage de l'inhibition comme symptôme pathologique particulier à l'inhibition comme symptôme universel de tout apprentissage, passage d'une souffrance individuelle, vécue comme impuissance, à un malheur humain relevant d'un savoir toujours en retrait de la vérité impossible. Gardant ainsi au symptôme son statut intrinsèque au sujet tout en ayant la fonction de fournir à l'enfant, à ce sujet, la possibilité de s'y reconnaître dans la stratégie qui lui est propre pour s'orienter dans l'existence, ce qui s'articule avec ce que nous nommons plus bas une «rectification de son rapport au savoir»

Il nous faut travailler dans cette double référence : la cognition, travail de remédiation, pour aider l'enfant en le désenlisant de sa « déficience », à supporter ce que la psychothérapie proprement dite va convoquer pour lui du côté d'une confrontation à la perte et au renoncement mais aussi et surtout au consentement au désir que ladite inhibition marque et masque tout à la fois. Si l'abord de la question de la perte est indirect, l'enfant peut faire d'une demande de psychothérapie $s a$ demande et témoigner ainsi d'une première rectification de son rapport au savoir.

\footnotetext{
18 Sur l'intérêt du dialogue entre psychanalyse et sciences cognitives, voir l'article récent de R. Le Roux, Psychanalyse et cybernétique. Les machines de Lacan, [12].
} 
Les symptômes scolaires actuels qui motivent les consultations nous sont presque tous présentés dans les registres de l'agitation, de l'hyperactivité, autrement dit, du côté du «trop» (trop d'activation/défaut d'inhibition). Ils font, de plus, quasiment office de «névrose actuelle» puisqu' «à part ça, tout va bien», renforçant de la sorte l'opacité des liens à effectuer avec l'organisation névrotique infantile du moins tel que cela peut apparaître du point de vue de l'autre, c'est-à-dire des enseignants ou des parents, mais l'enfant lui..., s'il a la chance de rencontrer un clinicien qui lui laisse la possibilité d'articuler son malaise dans le savoir à sa névrose infantile, alors se présentera peut-être l'espoir d'une véritable rectification subjective.

Enfin, dernier point d'appui dans cette proposition thérapeutique à double entrée, la prise en charge «éducative» au sens large, et psychothérapeutique proprement dite: il semble que, plus que le contenu de la démarche proposée, nécessairement propre à chaque champ, et heureusement, plus que ce contenu, importe la position du «thérapeute»; si le psy suppose de fait à l'enfant un savoir subjectif, le second lui présupposerait des compétences. De cette supposition conjointe dépend la remise en mouvement du désir et le bon usage des inhibitions.

C'est ce dont a témoigné le double engagement décrit dans notre exemple clinique : des allersretours sous forme de dialogues orthophoniste/psychanalyste semblent avoir dénoué des points d'impasse pour le petit garçon qui, à ce jour, a lâché l'oralité et la «crampe de l'écrivain ».

\section{Références}

[1] Houdé O. Rationalité, développement et inhibition : un nouveau cadre d'analyse. Paris: PUF; 1995.

[2] Freud S. Résultats, idées, problèmes II. Paris: PUF; 1985.

[3] Lacan J. Ecrits. Paris: Seuil; 1966.

[4] Winnicott DWW. Processus de maturation chez l'enfant. Paris: Payot; 1983.

[5] Freud S. Trois essais sur la théorie sexuelle (1962). [Trad. par B. Reverchon-Jouve]. Paris: Gallimard, coll. «Idées »; 1905.

[6] Houdé O. La Psychologie de l’enfant. 2e éd. Paris : PUF, coll. «Que sais-je ?»; 2005.

[7] Piaget J, Inhelder B. La psychologie de l'enfant. Paris: PUF; 1966.

[8] Lacan J. L'angoisse (1962-1963). Paris: Seuil; 2004.

[9] Freud S. Inhibition, symptôme et angoisse (1926). Paris : PUF, coll. «Quadrige»; 2002.

[10] Freud S. Naissance de la psychanalyse. Paris: PUF; 1973.

[11] Gori R. La passion de la causalité : une parole en cause. Clin med 1993;37/38:7-34.

[12] Le Roux R. Psychanalyse et cybernétique. Les machines de Lacan. Evol psychiatr 2007;72:346-69. 